J. Nonlinear Var. Anal. 5 (2021), No. 6, pp. 981-987

Available online at http://jnva.biemdas.com

https://doi.org/10.23952/jnva.5.2021.6.10

\title{
SOME HYPERGEOMETRIC TRANSFORMATIONS AND REDUCTION FORMULAS FOR THE GAUSS FUNCTION AND THEIR APPLICATIONS INVOLVING THE CLAUSEN FUNCTION
}

\author{
H.M. SRIVASTAVA ${ }^{1,2,3,4, *}$, M.I. QURESHI ${ }^{5}$, S.H. MALIK ${ }^{5}$ \\ ${ }^{1}$ Department of Mathematics and Statistics, University of Victoria, \\ Victoria, British Columbia V8W 3R4, Canada \\ ${ }^{2}$ Department of Medical Research, China Medical University Hospital, \\ China Medical University, Taichung 40402, Taiwan \\ ${ }^{3}$ Department of Mathematics and Informatics, Azerbaijan University, Baku AZ1007, Azerbaijan \\ ${ }^{4}$ Section of Mathematics, International Telematic University Uninettuno, I-00186 Rome, Italy \\ ${ }^{5}$ Department of Applied Sciences and Humanities, Faculty of Engineering and Technology, \\ Jamia Millia Islamia (A Central University), New Delhi 110025, India
}

\begin{abstract}
The aim of this paper is to obtain some closed forms of hypergeometric reduction formulas for the following Gauss functions ${ }_{2} F_{1}\left[\alpha, \alpha+\frac{1}{2} ; 2 \alpha-1 ; z\right]$ and ${ }_{2} F_{1}\left[\alpha-1, \alpha-\frac{3}{2} ; 2 \alpha-1 ; z\right]$, and the Clausen function: ${ }_{3} F_{2}\left[\gamma+1, \beta, \beta+\frac{1}{2} ; \gamma, 2 \beta ; z\right]$ by using the series rearrangement technique.

Keywords. Generalized hypergeometric functions; Hypergeometric transformations and reduction formulas; Gauss and Clausen hypergeometric functions.
\end{abstract}

\section{INTRODUCTION AND PRELIMINARIES}

In terms of the familiar (Euler's) Gamma function $\Gamma(z)$, which is defined, for $z \in \mathbb{C} \backslash \mathbb{Z}_{0}^{-}$, by

$$
\Gamma(z)= \begin{cases}\int_{0}^{\infty} e^{-t} t^{z-1} d t & (\mathfrak{R}(z)>0), \\ \frac{\Gamma(z+n)}{n-1} & \left(z \in \mathbb{C} \backslash \mathbb{Z}_{0}^{-} ; n \in \mathbb{N}\right), \\ \prod_{j=0}^{n}(z+j) & \end{cases}
$$

${ }^{*}$ Corresponding author.

E-Mail addresses: harimsri@math.uvic.ca (H. M. Srivastava), miqureshi_delhi@yahoo.co.in (M. I. Qureshi), malikshakir774@gmail.com (S.H. Malik).

Received June 15, 2021; Accepted July 15, 2021.

(C)2021 Journal of Nonlinear and Variational Analysis 
the general Pochhammer symbol (or the shifted factorial) $(\lambda)_{v},(\lambda, v \in \mathbb{C}$ ) is defined by (see, for example, $[1,2]$ )

$$
(\lambda)_{v}:=\frac{\Gamma(\lambda+v)}{\Gamma(\lambda)}= \begin{cases}1 & (v=0 ; \lambda \in \mathbb{C} \backslash\{0\}), \\ \prod_{j=0}^{n-1}(\lambda+j) & (v=n \in \mathbb{N} ; \lambda \in \mathbb{C}), \\ \frac{(-1)^{k} n !}{(n-k) !} & \left(\lambda=-n ; v=k ; n, k \in \mathbb{N}_{0} ; 0 \leqq k \leqq n\right), \\ 0 & \left(\lambda=-n ; v=k ; n, k \in \mathbb{N}_{0} ; k>n\right), \\ \frac{(-1)^{k}}{(1-\lambda)_{k}} & (v=-k ; k \in \mathbb{N} ; \lambda \in \mathbb{C} \backslash \mathbb{Z}),\end{cases}
$$

where it is understood conventionally that $(0)_{0}:=1$ and assumed tacitly that the Gamma quotient exists.

From now on, the following standard notations are used:

$$
\mathbb{N}:=\{1,2,3, \cdots\}, \mathbb{N}_{0}:=\mathbb{N} \cup\{0\} \quad \text { and } \quad \mathbb{Z}_{0}^{-}:=\mathbb{Z}^{-} \cup\{0\}=\{0,-1,-2, \cdots\} .
$$

Moreover, as usual, the symbols $\mathbb{C}, \mathbb{R}, \mathbb{N}, \mathbb{Z}, \mathbb{R}^{+}$, and $\mathbb{R}^{-}$denote the sets of complex numbers, real numbers, natural numbers, integers, positive, and negative real numbers, respectively.

The celebrated Gauss hypergeometric function, ${ }_{2} F_{1}$, the Kummer (or confluent) hypergeometric function, ${ }_{1} F_{1}$, the Clausen hypergeometric function ${ }_{3} F_{2}$, and various other mathematical functions of hypergeometric type, are all contained in the generalized hypergeometric function ${ }_{p} F_{q}$ involving $p$ numerator parameters, $a_{1}, \cdots, a_{p}$, and $q$ denominator parameters, $b_{1}, \cdots, b_{q}$, as special cases.

Following the standard notations and conventions, we define it here as follows:

$$
\begin{gathered}
{ }_{p} F_{q}\left[\begin{array}{c}
\left(\alpha_{p}\right) ; \\
\left(\beta_{q}\right) ;
\end{array}\right]={ }_{p} F_{q}\left[\begin{array}{c}
\alpha_{1}, \alpha_{2}, \cdots, \alpha_{p} ; \\
\beta_{1}, \beta_{2}, \cdots, \beta_{q} ;
\end{array}\right] \\
:=\sum_{n=0}^{\infty} \frac{\left(\alpha_{1}\right)_{n}\left(\alpha_{2}\right)_{n} \cdots\left(\alpha_{p}\right)_{n}}{\left(\beta_{1}\right)_{n}\left(\beta_{2}\right)_{n} \cdots\left(\beta_{q}\right)_{n}} \frac{z^{n}}{n !}, \\
\left(p, q \in \mathbb{N}_{0} ; p \leqq q+1 ; p \leqq q \text { and }|z|<\infty ; p=q+1 \text { and }|z|<1 ;\right. \\
p=q+1,|z|=1 \text { and } \mathfrak{R}(\omega)>0 ; \\
p=q+1,|z|=1(z \neq 1) \text { and }-1<\Re(\omega) \leqq 0),
\end{gathered}
$$

where, by convention, a product over an empty set is interpreted as 1 , and

$$
\begin{gathered}
\omega:=\sum_{j=1}^{q} \beta_{j}-\sum_{j=1}^{p} \alpha_{j} \\
\left(\alpha_{j} \in \mathbb{C}(j=1,2, \cdots, p) ; \beta_{j} \in \mathbb{C} \backslash \mathbb{Z}_{0}^{-}(j=1,2 . \cdots . q)\right),
\end{gathered}
$$

and $\mathfrak{R}(\omega)$ is the real part of complex number $\omega$ (see, for details, [1, 3, 4]). 
Remark 1.1. Throughout the rest of this paper, the applicable parametric and argument constraints, which would correspond appropriately to the above-mentioned parametric and argument constraints, will be tacitly assumed to be satisfied appropriately. Moreover, the exceptional values of the parameters and the arguments, which are involved in any equation, are also tacitly excluded.

The following results will be needed in this paper.

A Transformation Formula for the Gauss Function ${ }_{2} F_{1}$ (see [5, p. 112, Eq. (16)]; see also [6, p. 251, Eq. (9.6.4)]):

$$
\begin{aligned}
{ }_{2} F_{1}\left[\begin{array}{r}
a, a+\frac{1}{2} ; \\
b ;
\end{array}\right] & =(1-z)^{-a}{ }_{2} F_{1}\left[\begin{array}{rr}
2 a, 2 b-2 a-1 ; & \left.\frac{\sqrt{1-z}-1}{2 \sqrt{1-z}}\right] \\
b ; &
\end{array}\right. \\
& \left(|\arg (1-z)|<\pi ; b \in \mathbb{C} \backslash \mathbb{Z}_{0}^{-}\right) .
\end{aligned}
$$

Euler's Linear Transformation (see [6, p. 248, Eq. (9.5.3)]; see also [7, p.68, Eq.(2.2.7)]):

$$
\begin{gathered}
{ }_{2} F_{1}\left[\begin{array}{rr}
\beta, \gamma ; & \\
\delta ; & z
\end{array}\right]=(1-z)^{\delta-\beta-\gamma}{ }_{2} F_{1}\left[\begin{array}{r}
\delta-\beta, \delta-\gamma ; \\
\delta ;
\end{array}\right] \\
\left(|\arg (1-z)|<\pi ; \delta \in \mathbb{C} \backslash \mathbb{Z}_{0}^{-}\right) .
\end{gathered}
$$

The Binomial Expansion (see [1, p. 47]):

$$
{ }_{1} F_{0}\left[\begin{array}{c}
\lambda ; \\
-;
\end{array}\right]=(1-z)^{-\lambda} \quad(|z|<1 ; \lambda \in \mathbb{C}) .
$$

Two Closed Forms of the Gauss Function ${ }_{2} F_{1}$ (see [1, p. 70, Exercise 10]; see also [7, p. 185, Entry (39)], and [3, p. 19, Eq. (1.5.20)]):

$$
\begin{gathered}
{ }_{2} F_{1}\left[\begin{array}{r}
\lambda, \lambda-\frac{1}{2} ; \\
2 \lambda ;
\end{array}\right]=\left(\frac{2}{1+\sqrt{1-z}}\right)^{2 \lambda-1} \\
\left(|\arg (1-z)|<\pi ; 2 \lambda \in \mathbb{C} \backslash \mathbb{Z}_{0}^{-}\right)
\end{gathered}
$$

and

$$
\begin{gathered}
{ }_{2} F_{1}\left[\begin{array}{r}
\lambda, \lambda+\frac{1}{2} ; \\
2 \lambda ;
\end{array}\right]=\frac{1}{\sqrt{1-z}}\left(\frac{2}{1+\sqrt{1-z}}\right)^{2 \lambda-1} \\
\left(|\arg (1-z)|<\pi ; 2 \lambda \in \mathbb{C} \backslash \mathbb{Z}_{0}^{-}\right) .
\end{gathered}
$$

A Reduction Formula Recorded by Prudnikov et al. (see [8, p. 460, Entry (103)]):

$$
\begin{gathered}
{ }_{2} F_{1}\left[\begin{array}{c}
\alpha, \alpha+\frac{1}{2} ; \\
2 \alpha-1 ;
\end{array}\right] \stackrel{\circ}{=} \frac{1}{2 \alpha-1}[y+2(1-\alpha)(y-2)] y^{2 \alpha}(2-y)^{-3} \\
\left(z y^{2}=4(y-1) ; 2 \alpha-1 \in \mathbb{C} \backslash \mathbb{Z}_{0}^{-} ;|z|<1 ;\left|\frac{y-1}{y^{2}}\right|<\frac{1}{4}\right) .
\end{gathered}
$$


Remark 1.2. The symbol $\stackrel{\circ}{=}$ exhibits the fact that reduction formula (1.5) does not hold as stated. In fact, equation (1.5) has not been verified numerically. In this connection, see the corrected version (2.2) of the erroneous result (1.5).

The rest of this article is organized as follows. In Section 2, we state the closed forms of some reduction formulas for the Gauss function ${ }_{2} F_{1}(z)$ and the Clausen function ${ }_{3} F_{2}(z)$. In Section 3 , we present the derivations of the reduction formulas for the Gauss function. In Section 4, we apply our reduction formula (2.1) to the Clausen function by using the familiar series rearrangement technique (see, for details, [2, Chapter 2]).

\section{The Main Hypergeometric Reduction Formulas in Closed Forms}

In this section, we state our main hypergeometric reduction formulas for the Gauss function ${ }_{2} F_{1}$ and the Clausen function ${ }_{3} F_{2}$ in closed forms. As we already mentioned in Remark 1.1, any values of the parameters and arguments, which would lead to the results that do not make sense, are tacitly excluded.

$$
\begin{aligned}
& { }_{2} F_{1}\left[\begin{array}{c}
\alpha, \alpha+\frac{1}{2} ; \\
2 \alpha-1 ;
\end{array}\right]=\left(\frac{1+\sqrt{1-z}}{2}\right)^{2(1-\alpha)}\left(\frac{1+2(\alpha-1) \sqrt{1-z}}{(2 \alpha-1)(1-z)^{\frac{3}{2}}}\right) \\
& \left(|\arg (1-z)|<\pi ; 2 \alpha-1 \in \mathbb{C} \backslash \mathbb{Z}_{0}^{-}\right) . \\
& { }_{2} F_{1}\left[\begin{array}{cc}
\alpha, \alpha+\frac{1}{2} ; & 4 z-4 \\
2 \alpha-1 ; & z^{2}
\end{array}\right]=\frac{(z-1)^{2(1-\alpha)}}{2 \alpha-1}[2(1-\alpha)(z-2)-z] \frac{z^{2 \alpha}}{(2-z)^{3}} \\
& \left(\left|\frac{z-1}{z^{2}}\right|<\frac{1}{4} ; 2 \alpha-1 \in \mathbb{C} \backslash \mathbb{Z}_{0}^{-} ; \mathfrak{R}(z)>2\right) . \\
& { }_{2} F_{1}\left[\begin{array}{r}
\alpha-1, \alpha-\frac{3}{2} ; \\
2 \alpha-1 ;
\end{array}\right]=\left(\frac{1+\sqrt{1-z}}{2}\right)^{2(1-\alpha)}\left(\frac{1+2(\alpha-1) \sqrt{1-z}}{(2 \alpha-1)}\right) \\
& \left(|\arg (1-z)|<\pi ; 2 \alpha-1 \in \mathbb{C} \backslash \mathbb{Z}_{0}^{-}\right) .
\end{aligned}
$$

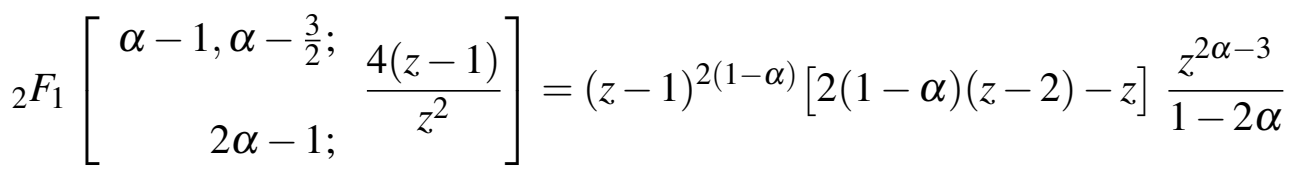

$$
\begin{aligned}
& \left(\left|\frac{z-1}{z^{2}}\right|<\frac{1}{4} ; 2 \alpha-1 \in \mathbb{C} \backslash \mathbb{Z}_{0}^{-} ; \Re(z) \geqq 2\right) \text {. } \\
& { }_{3} F_{2}\left[\begin{array}{r}
\gamma+1, \beta, \beta+\frac{1}{2} ; \\
\gamma, 2 \beta ;
\end{array}\right]=\left(\frac{2}{1+\sqrt{1-z}}\right)^{2 \beta-1} \\
& \left(\frac{2 \gamma(1+\sqrt{1-z})+z(2 \beta-4 \gamma+(1-2 \gamma) \sqrt{1-z})+2(\gamma-\beta) z^{2}}{2 \gamma(1-z)^{\frac{3}{2}}(1-z+\sqrt{1-z})}\right)
\end{aligned}
$$




$$
\left(|\arg (1-z)|<\pi ; \gamma, 2 \beta \in \mathbb{C} \backslash \mathbb{Z}_{0}^{-}\right) .
$$

Remark 2.1. We have successfully verified each of the reduction formulas (2.1), (2.2), (2.3), (2.4) and (2.5) numerically.

\section{Demonstration of the Reduction Formulas (2.1) to (2.4)}

This section is devoted to the derivations of the first four of our main results (2.1) to (2.5).

Proof of the reduction formulas (2.1) and (2.2):

Using transformation (1.1) on the left-hand side of reduction formula (2.1), we obtain

$$
{ }_{2} F_{1}\left[\begin{array}{c}
\alpha, \alpha+\frac{1}{2} ; \\
2 \alpha-1 ;
\end{array}\right]=(1-z)^{-\alpha}{ }_{2} F_{1}\left[\begin{array}{rr}
2 \alpha, 2 \alpha-3 ; & \frac{\sqrt{1-z}-1}{2 \sqrt{1-z}}
\end{array}\right]=:(1-z)^{-\alpha} \Phi(z),
$$

where

$$
\begin{aligned}
& \Phi(z):={ }_{2} F_{1}\left[\begin{array}{rr}
2 \alpha, 2 \alpha-3 ; & \frac{\sqrt{1-z}-1}{2 \sqrt{1-z}} \\
2 \alpha-1 ; &
\end{array}\right] \\
& =\sum_{r=0}^{\infty} \frac{(2 \alpha)_{r}(2 \alpha-3)_{r}}{r !(2 \alpha-1)_{r}}\left(\frac{\sqrt{1-z}-1}{2 \sqrt{1-z}}\right)^{r} \\
& =\sum_{r=0}^{\infty} \frac{(2 \alpha-3)_{r}}{r !}\left(1+\frac{r}{2 \alpha-1}\right)\left(\frac{\sqrt{1-z}-1}{2 \sqrt{1-z}}\right)^{r}
\end{aligned}
$$

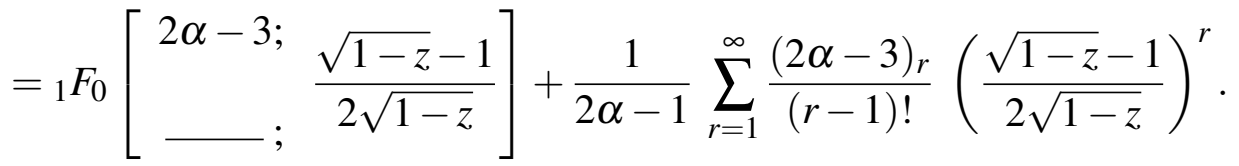

If we replace $r$ by $r+1$ in the second member of the right-hand side of equation (3.2), we obtain

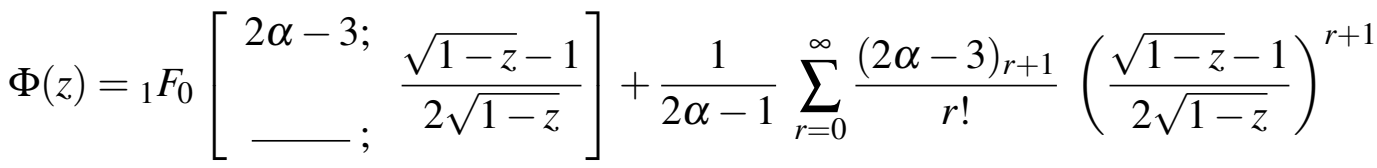

$$
\begin{aligned}
& ={ }_{1} F_{0}\left[\begin{array}{rr}
2 \alpha-3 ; & \frac{\sqrt{1-z}-1}{2 \sqrt{1-z}}
\end{array}\right] \\
& +\left(\frac{2 \alpha-3}{2(2 \alpha-1)}\right)\left(\frac{\sqrt{1-z}-1}{\sqrt{1-z}}\right) \sum_{r=0}^{\infty} \frac{(2 \alpha-2)_{r}}{r !}\left(\frac{\sqrt{1-z}-1}{2 \sqrt{1-z}}\right)^{r} \\
& ={ }_{1} F_{0}\left[\begin{array}{rr}
2 \alpha-3 ; & \frac{\sqrt{1-z}-1}{2 \sqrt{1-z}}
\end{array}\right] \\
& +\left(\frac{2 \alpha-3}{2(2 \alpha-1)}\right)\left(\frac{\sqrt{1-z}-1}{\sqrt{1-z}}\right){ }_{1} F_{0}\left[\begin{array}{rr}
2 \alpha-2 ; & \frac{\sqrt{1-z}-1}{2 \sqrt{1-z}}
\end{array}\right] \text {. }
\end{aligned}
$$


Now, by applying binomial expansion (1.3) in the right-hand side of equation (3.3), we obtain

$$
\Phi(z)=\left(\frac{1+\sqrt{1-z}}{2 \sqrt{1-z}}\right)^{2(1-\alpha)}\left(\frac{1+2(\alpha-1) \sqrt{1-z}}{(2 \alpha-1) \sqrt{1-z}}\right)
$$

Finally, we substitute the value of $\Phi(z)$ from this last equation (3.4) into the right-hand side of equation (3.1). After some simplification, we obtain the right-hand side of reduction formula (2.1).

Upon setting $z \mapsto \frac{4(z-1)}{z^{2}}$ in both sides of equation (2.1), and after simplification, we are led to equation (2.2), which is the correct form of the erroneous result (1.5).

Proof of the reduction formulas (2.3) and (2.4):

Using Euler's linear transformation (1.2) in the left-hand side of equation (2.1), we obtain the required reduction formula (2.3).

If we use Euler's linear transformation (1.2) in the left-hand side of equation (2.2), we obtain the required result (2.4).

\section{The Application of (2.1) Involving the Clausen Function}

In this section, we apply our first reduction formula (2.1) in the derivation of the reduction formula (2.5) involving the Clausenian hypergeometric function ${ }_{3} F_{2}$.

Proof of the reduction formula (2.5):

For convenience, we denote the first member of the reduction formula by $\Psi(z)$, so that

$$
\begin{aligned}
\Psi(z) & :={ }_{3} F_{2}\left[\begin{array}{r}
\gamma+1, \beta, \beta+\frac{1}{2} ; \\
\gamma, 2 \beta ;
\end{array}\right]=\sum_{r=0}^{\infty} \frac{(\beta)_{r}\left(\beta+\frac{1}{2}\right)_{r}}{(2 \beta)_{r}}\left(1+\frac{r}{\gamma}\right) \frac{z^{r}}{r !} \\
& =\sum_{r=0}^{\infty} \frac{(\beta)_{r}\left(\beta+\frac{1}{2}\right)_{r}}{(2 \beta)_{r}} \frac{z^{r}}{r !}+\frac{1}{\gamma} \sum_{r=1}^{\infty} \frac{(\beta)_{r}\left(\beta+\frac{1}{2}\right)_{r}}{(2 \beta)_{r}} \frac{z^{r}}{(r-1) !} \\
& ={ }_{2} F_{1}\left[\begin{array}{c}
\beta, \beta+\frac{1}{2} ; \\
2 \beta ;
\end{array}\right]+\frac{1}{\gamma} \sum_{r=1}^{\infty} \frac{(\beta)_{r}\left(\beta+\frac{1}{2}\right)_{r}}{(2 \beta)_{r}} \frac{z^{r}}{(r-1) !} .
\end{aligned}
$$

We now replace $r$ by $r+1$ in the series occurring on the right-hand side of the last member of equation (4.1). We thus find that

$$
\begin{aligned}
\Psi(z) & ={ }_{2} F_{1}\left[\begin{array}{r}
\beta, \beta+\frac{1}{2} ; \\
2 \beta ;
\end{array}\right]+\frac{1}{\gamma} \sum_{r=0}^{\infty} \frac{(\beta)_{r+1}\left(\beta+\frac{1}{2}\right)_{r+1}}{(2 \beta)_{r+1}} \frac{z^{r+1}}{r !} \\
& ={ }_{2} F_{1}\left[\begin{array}{r}
\beta, \beta+\frac{1}{2} ; \\
2 \beta ;
\end{array}\right]+\frac{\beta\left(\beta+\frac{1}{2}\right) z}{2 \beta \gamma} \sum_{r=0}^{\infty} \frac{(\beta+1)_{r}\left(\beta+\frac{3}{2}\right)_{r}}{(2 \beta+1)_{r}} \frac{z^{r}}{r !} \\
& ={ }_{2} F_{1}\left[\begin{array}{c}
\beta, \beta+\frac{1}{2} ; \\
2 \beta ;
\end{array}\right]+\frac{(2 \beta+1) z}{4 \gamma}{ }_{2} F_{1}\left[\begin{array}{c}
\beta+1, \beta+\frac{3}{2} ; \\
2 \beta+1 ;
\end{array}\right] .
\end{aligned}
$$


This last expression in (4.2) for $\Psi(z)$ readily yields the right-hand side of the required reduction formula (2.5) when we make use of the closed form (1.4) in the first term and also our reduction formula (2.1) in the second term.

\section{CONCLUding REMARKS AND OBSERVATIONS}

We conclude our present investigation by observing that several interesting and potentially useful closed-form reduction formulas can be obtained in an analogous manner. Moreover, the hypergeometric reduction formulas, which we have derived in this paper in closed form, are expected to have useful applications in a wide range of problems in the mathematical, physical, and statistical sciences (see, for example, [9]; see also the recent works [10, 11, 12, 13, 14, 15] dealing extensively with the methodology and techniques based upon Gauss, Clausen and related hypergeometric functions in one and two variables).

\section{REFERENCES}

[1] E.D. Rainville, Special Functions, The Macmillan Company, New York 1960; Reprinted by Chelsea Publishing Company, Bronx, New York, 1971.

[2] H.M. Srivastava, H.L. Manocha, A Treatise on Generating Functions, Halsted Press (Ellis Horwood Limited, Chichester), John Wiley and Sons, New York, Chichester, Brisbane and Toronto, 1984.

[3] L.J. Slater, Generalized Hypergeometric Functions, Cambridge University Press, Cambridge, London and New York, 1966.

[4] H.M. Srivastava, P.W. Karlsson, Multiple Gaussian Hypergeometric Series, Ellis Horwood Series on Mathematics and Its Applications, Halsted Press (Ellis Horwood Limited, Chichester) John Wiley and Sons, New York, Chichester, Brisbane and Toronto, 1985.

[5] A. Erdélyi, W. Magnus, F. Oberhettinger, F.G. Tricomi, Higher Transcendental Functions, Vol. I, McGrawHill Book Company, New York, Toronto and London, 1953.

[6] N.N. Lebedev, Special Functions and Their Applications (Translated from the Russian by Richard A. Silverman), Prentice-Hall Incorporated, Englewood Cliffs, New Jersey, 1965.

[7] G.E. Andrews, R. Askey, R. Roy, Special Functions, Cambridge University Press, Cambridge, London and New York, 1999.

[8] A.P. Prudnikov, Yu. A. Brychkov, O.I. Marichev, Integrals and Series, Vol.3: More Special Functions, Nauka, Moscow, 1986 (In Russian); Translated from the Russian by G.G. Gould, Gordon and Breach Science Publishers, New York, Philadelphia, London, Paris, Montreux, Tokyo, Melbourne, 1990.

[9] H.M. Srivastava, B.R.K. Kashyap, Special Functions in Queuing Theory and Related Stochastic Processes, Academic Press, New York and London, 1982.

[10] T. Ergashev, A. Hasanov, Appell hypergeometric function and its application to the potential theory for a generalized bi-axially symmetric elliptic equation, Appl. Anal. Optim. 5 (2021), 57-76.

[11] H.M. Srivastava, M.I. Qureshi, S.A. Dar, Some novel Laplace-transform based integrals via hypergeometric techniques, Appl. Math. Inform. Sci. 14 (2020), 743-754.

[12] H.M. Srivastava, M.I. Qureshi, S. Jabee, Some general series identities and summation theorems for the Gauss hypergeometric function with negative integer numerator and denominator parameters, J. Nonlinear Convex Anal. 21 (2020), 463-478.

[13] H.M. Srivastava, M.I. Qureshi, S. Jabee, Some general series identities and summation theorems for Clausen's hypergeometric function with negative integer numerator and denominator parameters, J. Nonlinear Convex Anal. 21 (2020), 805-819.

[14] H.M. Srivastava, M.A. Shpot, The Clausenian hypergeomeric function ${ }_{3} F_{2}$ with unit argument and negative parameter differences, Appl. Math. Comput. 259 (2015), 819-827.

[15] H.M. Srivastava, Y. Vyas, K. Fatawat, Extensions of the classical theorems for very well-poised hypergeometric functions, Rev. Real Acad. Cienc. Exactas Fís. Natur. Ser. A Mat. RACSAM 113 (2019), 367-397. 